
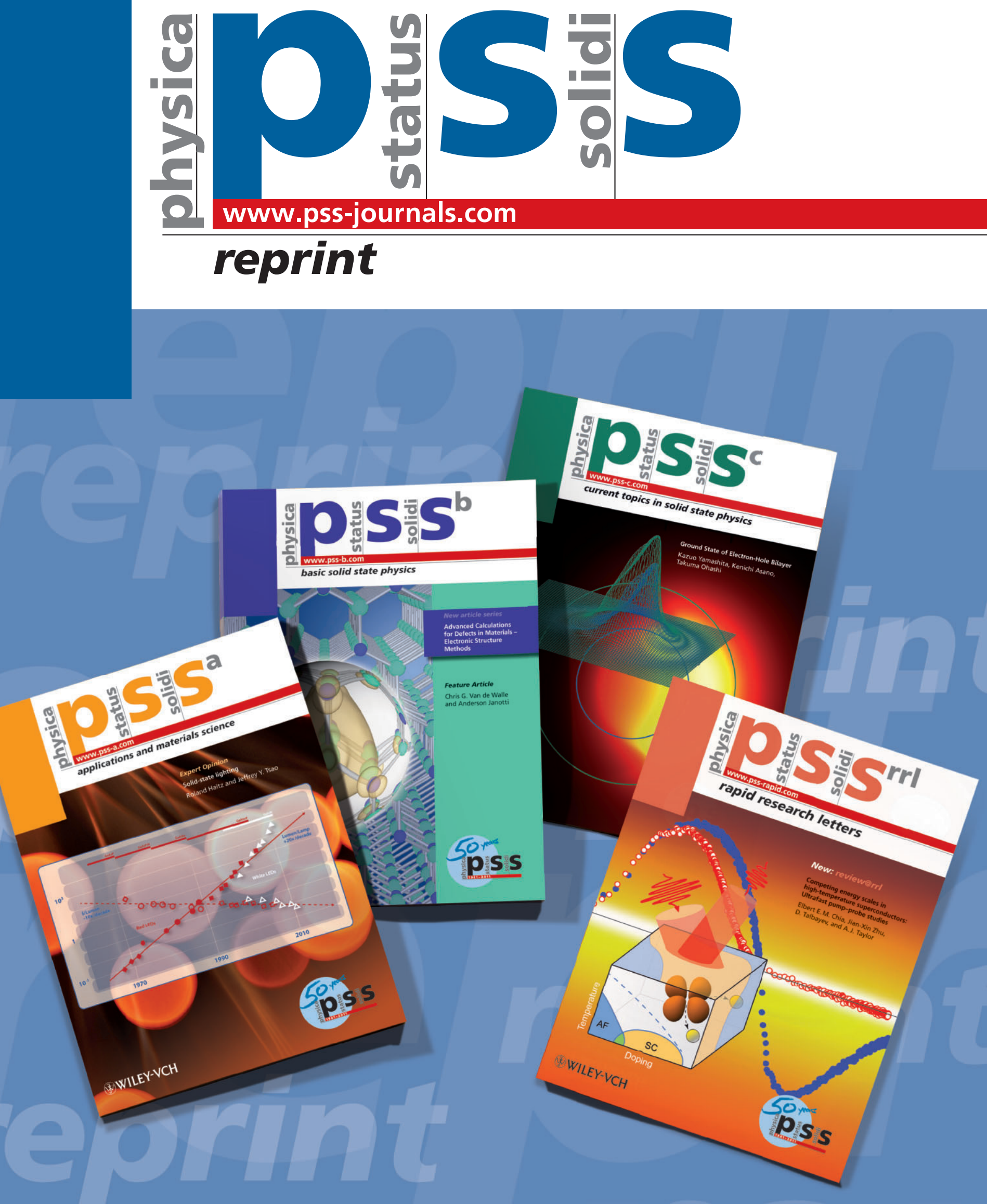


\title{
Plasmonic light-trapping in a-Si:H solar cells by front-side Ag nanoparticle arrays: A benchmarking study
}

\author{
F. O. Lenzmann ${ }^{*, 1}$, M. C. van Lare ${ }^{* *, 2}$, J. Salpakari', P. Spinelli', J. B. Notta', M. Dörenkämper', N. J. Bakker', \\ A. W. Weeber', and A. Polman ${ }^{2}$ \\ ${ }^{1}$ ECN Solar Energy, P.O. Box 11755 ZG Petten, The Netherlands \\ ${ }^{2}$ Center for Nanophotonics, FOM-Institute AMOLF, Science Park 104, 1098 XG Amsterdam, The Netherlands
}

Received 28 November 2012, revised 6 February 2013, accepted 27 February 2013

Published online 29 July 2013

Keywords a-Si:H solar cells, benchmarking, light-trapping, plasmonics

*Corresponding author: e-mail lenzmann@ecn.nl, Phone: +31 224564838, Fax: +31 224568214

***e-mail lare@amolf.nl, Phone: +31 207547100, Fax: +31 207547198

A series of (n-i-p) a-Si:H solar cells with light-trapping by front-side plasmonic Ag nanoparticle arrays was compared to a reference without the plasmonic arrays as well as to a benchmark with a conventional textured back-side reflector for light-trapping. The external quantum efficiency of the solar cells was determined experimentally by spectral response measurements. The comparison gives a comprehensive snap- shot of the potential of front-side plasmonic light-trapping in aSi:H solar cells for the array parameterization used in this study. Relative to the reference the plasmonic arrays lead to clearly enhanced light-trapping in the longer wavelength range (600$800 \mathrm{~nm}$ ). This enhancement is lower than the one achieved by the benchmark though, which is discussed in terms of further research perspectives.
1 Introduction Plasmonic effects are optical phenomena arising from the interaction of free electrons in metallic conductors with light. Since these phenomena allow the manipulation of the directionality of light, plasmon optics can be applied for light-trapping and antireflection purposes in solar cells. Light-trapping is crucial in solar cells, in particular those based on amorphous and microcrystalline silicon thin films due to the unfavorable charge carrier mobilities and lifetimes in these materials. State-of-the-art light-trapping concepts rely on the texturing of transparent conductive oxide (TCO) layers in these cells, leading to efficient path length enhancement of the incident sunlight in the thin films by light-scattering [1].

The application perspectives for the new light-trapping concepts based on plasmon optics are currently increasingly explored. They hold promise in that the light-trapping limits applicable to state-of-the-art concepts can be surpassed by plasmonic approaches [2] and in terms of their high degree of versatility. Plasmonic light-trapping is expected to enable extremely thin solar cells to achieve efficiencies rivalling or surpassing those of the thicker solar cells manufactured today (leading to cost savings). A systematic overview of the various concepts, design rules and perspectives of plasmonic light-trapping in solar cells is given in a recent review paper [3].

Among the most promising results achieved thus far, is the fabrication of an only $90 \mathrm{~nm}$ thick a-Si:H solar cell with an efficiency of $9.6 \%$ based on light-trapping by surface plasmon polaritons at the back-contact [4]. This is an example of a back-side-configuration which has the fundamental advantage of avoiding Fano resonance losses $[5,6]$ and receives therefore increasing attention.

Nevertheless, due to the relatively straightforward integration, the most abundantly explored concept is lightscattering by metal nanoparticles at the front surface of solar cells (=side of light incidence). In this configuration, where the particles are situated at the interface of two dielectrics with different permittivity, light is scattered with a high degree of selectivity into the medium of the higher permittivity. If this medium is the absorber layer of a solar cell, it will result in enhanced light-trapping, since the scattered light acquires an angular spread that increases its 
optical path length in the absorber layer [3]. Numerous studies have been published in recent years documenting the enhanced light-trapping arising from front-side plasmonic arrays in solar cells (e.g., [5-9]). It should be noted that these studies typically assess the enhanced lighttrapping by comparison with reference devices that differ from the plasmonic solar cells only in the absence of the plasmonic particles. These reference devices are indeed most appropriate for scrutinizing the fundamental aspects of the associated plasmon optics. However, they are in general configured without (optimized) conventional light-trapping schemes and thus do not provide a comparison of the plasmonic concepts to state-of-the-art light-trapping benchmarks.

Benchmarking studies are therefore needed in order to properly assess the full application potential of plasmon optics in solar cells. The present paper addresses this need by the photoelectric characterization of a-Si:H solar cells where the light-trapping of two differently configured plasmonic arrays of $\mathrm{Ag}$ nanoparticles is compared not only to a reference but also to a benchmark device with a textured back-side reflector.

\section{Experimental}

2.1 Methods The methods for the preparation of the solar cells have been described in earlier publications. In short, the various layers of the a-Si:H cells were fabricated by sputtering $(\mathrm{Ag} / \mathrm{ZnO}$ back-reflector, ITO antireflection layer), plasma enhanced chemical vapor deposition (a-Si:H silicon layers) and thermal evaporation (Ag front-grid) on $10 \times 2.5 \mathrm{~cm}^{2}$ sized glass substrates [10]. The plasmonic Ag nanocylinder arrays were fabricated by substrate conformal imprint lithography (SCIL) as described in Ref. [5].

The external quantum efficiency (EQE) of the solar cells was measured with an Optosolar SR300 setup equipped with a Jobin Yvon iHR320 monochromator. The setup was calibrated with a crystalline silicon reference solar cell and the measurements were carried out with a wavelength resolution of $10 \mathrm{~nm}$.

2.2 Solar cell designs Illustrations of the four different solar cells are shown in Fig. 1. Cells b and $\mathrm{c}$ are plasmonic solar cells with $\mathrm{Ag}$ nanoparticle arrays at the front-side. Cell a serves as reference (which differs from plasmonic cell $b$ only in the absence of the Ag nanoparticle array) and cell $d$ is the benchmark device with a textured back-side reflector.

The active layer of all four cells is a $\sim 380 \mathrm{~nm}$ thick $\mathrm{n}-\mathrm{i}-\mathrm{p}$ stack of a-Si:H layers and the back reflector consists of a layer stack of $300 \mathrm{~nm} \mathrm{Ag}$ and $80 \mathrm{~nm} \mathrm{ZnO:Al.} \mathrm{The}$ differences between the four cells are: the presence or absence of a plasmonic layer, the use of a flat or a textured substrate and the thickness of the ITO layer (see Table 1). The variation of the ITO layer thickness was done on the basis of optical simulations which predict that in combination with the plasmonic arrays there is an optimum for the ITO layer thickness around $50 \mathrm{~nm}$ [11].
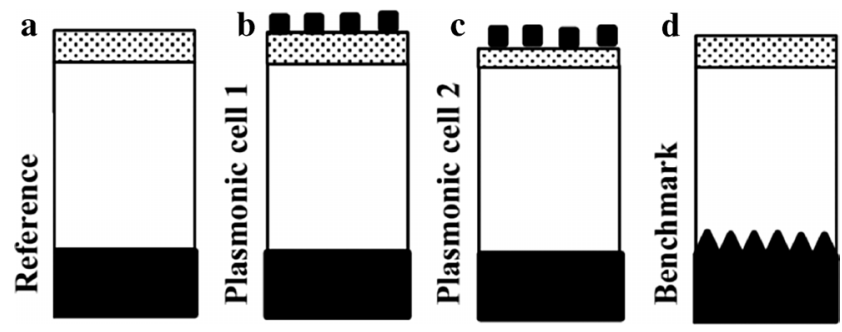

Figure 1 Illustrations showing schematically the various thin layers of the four solar cells compared in this study $(\mathrm{a}-\mathrm{d})$. These are (from bottom to top): (a) $\mathrm{Ag} / \mathrm{ZnO}$ :Al back reflector layer (in black), the $n-\mathrm{i}-\mathrm{p}$ a-Si:H stack (in white) and an ITO antireflection layer (dotted). Samples (b) and (c) comprise a plasmonic Ag nanocylinder array on top of the ITO layer (black squares). The back reflector layer is deposited on top of a flat glass substrate for samples $(\mathrm{a}-\mathrm{c})$. For sample (d) it is deposited on top of a textured glass substrate $\left(\mathrm{SnO}_{2}\right.$ coated glass, "Asahi U", in which the texture is defined by the surface structure of the $\mathrm{SnO}_{2}$ layer).

2.3 Images of the test samples The photograph of one of the solar cells in Fig. 2 shows the "macroscopic geometry" of the $10 \times 2.5 \mathrm{~cm}^{2}$ test samples. The samples are subdivided into many smaller and larger solar cells, which can all be contacted individually. This allows to print plasmonic Ag arrays on top of some of those cells and to keep others on the same substrate free of the arrays (thus allowing a very good comparability for cells which differ only in the presence or absence of the plasmonic array).

It consists of $\mathrm{Ag}$ nano-cylinders with a diameter of $200 \mathrm{~nm}$ which are arranged in a square pattern of $450 \mathrm{~nm}$ pitch. In the inset a larger area of the array is visible showing the perfect regularity of the pattern. The height of the cylinders was determined to be $80 \mathrm{~nm}$ by AFM measurements. This design was chosen on the basis of optical simulations similar to those published in Ref. [12]. The SEM image in Fig. 3 shows the geometry of the plasmonic $\mathrm{Ag}$ array.

\section{Results and discussion}

3.1 Plasmonic solar cells compared with the reference and the benchmark The EQE spectra of the four solar cells are shown in Fig. 4. Compared to the flat reference cell (dotted line), both plasmonic solar cells (gray and black continuous lines) show a clearly enhanced red response due to the presence of distinct peaks in the spectral

Table 1 Parameters in which the four solar cells differ.

\begin{tabular}{llll}
\hline solar cell & plasmonic array & substrate & $\begin{array}{l}\text { ITO } \\
\text { thickness } \\
(\mathrm{nm})\end{array}$ \\
\hline $\mathrm{a}=$ reference & no & flat & 80 \\
$\mathrm{~b}=$ plasmonic cell 1 & yes & flat & 80 \\
$\mathrm{c}=$ plasmonic cell 2 & yes & flat & 50 \\
$\mathrm{~d}=$ benchmark (Asahi) & no & textured & 80 \\
\hline
\end{tabular}




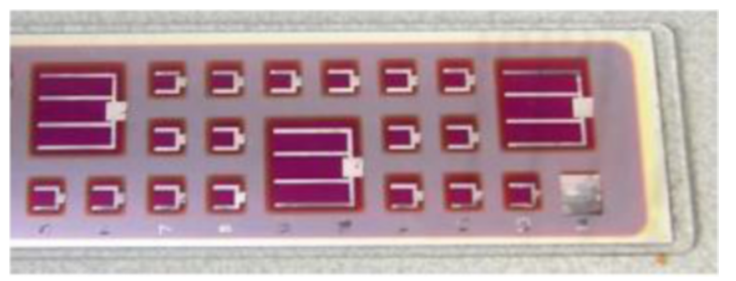

Figure 2 Photograph of a test device with its subdivisions into many smaller and larger solar cells.

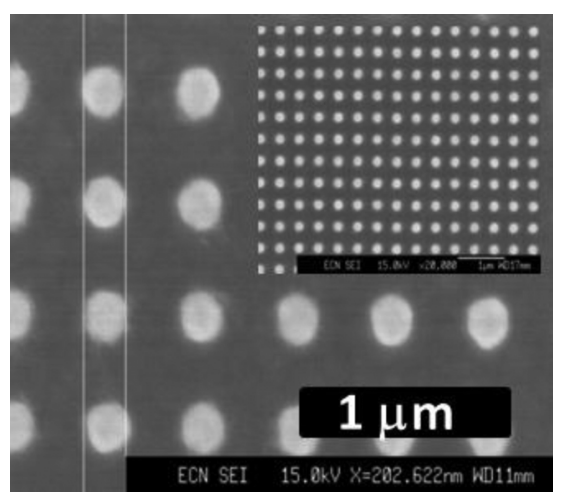

Figure 3 SEM image of the plasmonic array of Ag nano-cylinders on top of an a-Si:H solar cell. The geometric parameters of the nano-cylinder array are: diameter $=200 \mathrm{~nm}$, height $=80 \mathrm{~nm}$, pitch $=450 \mathrm{~nm}$.

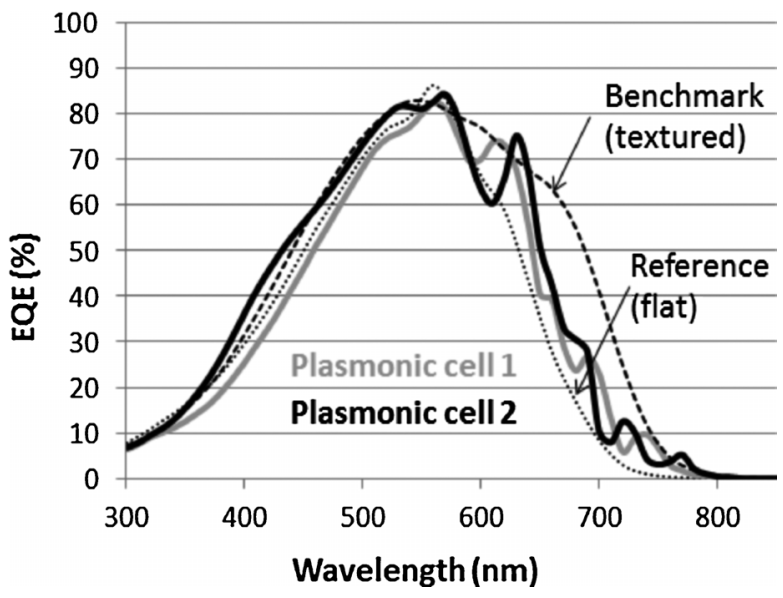

Figure $4 \mathrm{EQE}$ spectra of the four a-Si:H solar cells compared in this study. The characteristic differences in the spectral fingerprints are discussed in the text.

range between 600 and $800 \mathrm{~nm}$. These peaks rise well above the EQE values of the reference cell and can be assigned to the in-coupling of light to distinct waveguide modes of the thin a-Si:H layer as shown by us recently [6].

In the spectral range between 300 and $550 \mathrm{~nm}$ plasmonic cell 1 shows a slightly reduced response and plasmonic cell 2 shows a slightly enhanced response. The reduced response for cell 1 is (primarily) a consequence of Fano resonances,

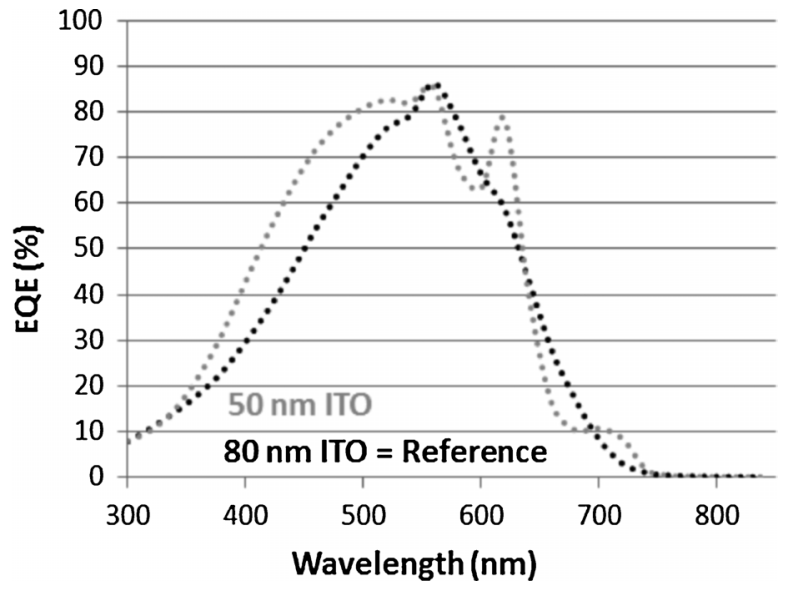

Figure 5 EQE spectrum of the reference cell with an $80 \mathrm{~nm}$ ITO layer (black dotted line) compared with a cell that differs from the reference only in the thickness of the ITO layer $(50 \mathrm{~nm})$, i.e., both cells are without plasmonic arrays.

$[5,6]$. The enhancement for cell 2 is not related to the plasmonic array. Instead, it results from the thinner ITO layer $(50 \mathrm{~nm}$ vs. $80 \mathrm{~nm})$, which shifts the maximum of the interference antireflection effect of the ITO layer to shorter wavelengths and therefore enhances the $\mathrm{EQE}$ in this spectral range (Fig. 5). It is only thanks to this effect and despite the Fano resonance losses that plasmonic cell 2 shows a slightly better response in the $300-550 \mathrm{~nm}$ range than the reference cell.

Compared to the textured benchmark cell (broken line) however, both plasmonic cells show significantly lower EQE values, most importantly, in the range between 600 and $800 \mathrm{~nm}$. Here the benchmark configuration shows a large and continuous broadband enhancement with respect to the planar reference device. The plasmonic devices on the other hand show distinct peaks most of which are also lower in intensity than the "envelope" defined by the benchmark response. In the range between 300 and $550 \mathrm{~nm}$ the differences with respect to the benchmark are less pronounced. Plasmonic cell 2 shows a marginally higher response than the benchmark cell in this range due to the thinner ITO layer as argued above. Plasmonic cell 1 shows a lower response in this range.

Within the boundary conditions of this experiment the coupling to distinct waveguide modes (in the plasmonic solar cells) clearly leads to a less effective enhancement of the total EQE than the random scattering of the benchmark.

As shown in our earlier work on the basis of optical simulations in Ref. [6], the optimization of the nano-cylinder diameter can lead to further significant improvements of the absorption spectrum in the $300-550 \mathrm{~nm}$ wavelength range. However, improvements in the 300-550 nm range alone are not expected to be sufficient to obtain overall absorption enhancements comparable to or better than the Asahi benchmark. As pointed out above in the discussion of Fig. 4 it is first and foremost the $600-800 \mathrm{~nm}$ range where the 


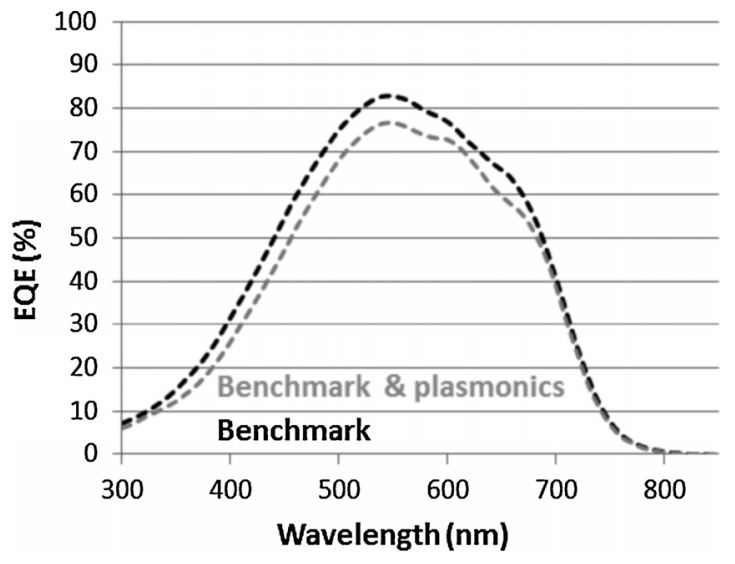

Figure $6 \mathrm{EQE}$ spectra of the benchmark device before and after the application of a plasmonic Ag array (black line = before, gray line $=$ after $)$.

benchmark outperforms the plasmonic cells (with the array configurations used in this present study). Also in this latter wavelength range additional improvements are therefore desirable. Corresponding investigations will be carried out in future work by addressing combined variations in the parameterization of the plasmonic arrays (next to the nanocylinder diameter, also height and pitch as well as the exchange of $\mathrm{Ag}$ by another plasmonic material).

\subsection{Combination of the benchmark device with} a plasmonic array A 5th type of solar cell device was fabricated in which a plasmonic Ag array was applied on top of the benchmark cell. In the following we refer to this device as "B\&P" cell (for Benchmark \& Plasmonic). Unlike for the solar cells with a flat back reflector, the combination of the random roughness (requiring a larger unit cell) and an array of metal nanoparticles, made it impossible to simulate this structure. A rationalization of the results shown in Fig. 6 in terms of optical modeling can thus not be given.

As can be seen in the figure, the EQE of the B\&P cell remains below that of the benchmark device throughout the complete spectral range. In this device configuration the loss channels associated with the plasmonic arrays, i.e., Fano resonances and parasitic absorption by the $\mathrm{Ag}$ nanocylinders are apparently dominant. We note here, that due to the impossibility to apply predictive optical simulations for this structure, the Ag array geometry may not be optimal at all. There may thus be other array geometries that enhance the EQE with respect to the benchmark cell.
4 Conclusions and Outlook The EQE of n-i-p a-Si: $\mathrm{H}$ solar cells with light-trapping by front-side plasmonic $\mathrm{Ag}$ arrays was measured and compared to a reference (without the Ag arrays) as well as to a benchmark with a textured back-side reflector for light-trapping. The EQE spectra show that the plasmonic solar cells outperform the reference device due to enhanced light-trapping. However, the best performance and thus most effective light-trapping is obtained with the benchmark device. Significant further improvements are thus needed for this front-side plasmonic concept to challenge the benchmark configuration. Optical simulation results published by us earlier [6] suggest that increased light-trapping can indeed be expected in the 300$550 \mathrm{~nm}$ wavelength range by the use of Ag nano-cylinders with a smaller diameter than applied in the present study. The experimental validation of these simulation results as well as the investigation of improvement potentials for the important $600-800 \mathrm{~nm}$ range will be the focus of future work.

Acknowledgements For technical support in the structural characterization of the samples by electron microscopy and atomic force microscopy, we are grateful to Erik Schuring (ECN). Work at AMOLF is part of the research program of FOM which is financially supported by NWO. Funding was provided by the Dutch NanoNextNL program under contract \# 02A.01

\section{References}

[1] J. Müller, B. Rech, J. Springer, and M. Vanecec, Solar Energy 77, 917 (2004).

[2] Z. Yu, A. Raman, and S. Fui, Proc Natl Acad Sci USA 107/41, 17491 (2010).

[3] H. A. Atwater and A. Polman, Nature Mater. 9, 205 (2010).

[4] V. E. Ferry, M. A. Verschuuren, M. C. van Lare, R. E. I. Schropp, H. A. Atwater, and A. Polman, Nano Lett. 11, 4239 (2011).

[5] P. Spinelli, M. Hebbink, R. de Waele, L. Black, F. Lenzmann, and A. Polman, Nano Lett. 11, 1760 (2011).

[6] M. C. van Lare, F. Lenzmann, M. A. Verschuuren, and A. Polman, Appl. Phys. Lett. 101, 221110 (2012).

[7] D. Derkacs, S. H. Lim, P. Matheu, W. Mar, and E. T. Yu, Appl. Phys. Lett. 89/9, 093103 (2006).

[8] S. Pillai, K. R. Catchpole, T. Trupke, and M. A. Green, J. Appl. Phys. 101/9, 093105 (2007).

[9] P. Matheu, S. H. Lim, D. Derkacs, C. McPheeters, and E. T. Yu, Appl. Phys. Lett. 93/11, 113108 (2008).

[10] W. J. Soppe, H. Borg, B. B. van Aken, C. Devilee, M. Dörenkämper, M. Goris, M. C. R. Heijna, J. Löffler, and P. Peeters, J. Nanosci. Nanotechnol. 11, 1 (2011).

[11] M. C. van Lare, unpublished results.

[12] P. Spinelli, V. E. Ferry, J. van de Groep, M. van Lare, M. A. Verschuuren, R. E. I. Schropp, H. A. Atwater, and A. Polman, J. Opt. 14, 024002 (2012). 